\title{
Continuer à s'engager dans des loisirs après avoir été infecté au VIH : entre quête de normalité et prescription sociale
}

Sylvain Ferez, Estelle Marin-Duval, Julie Thomas, Stéphane Héas \& Patrick Fougeyrollas

Remerciements : Cet article a été réalisé grâce à des données recueillies dans le cadre de deux enquêtes : une enquête nationale sur l'accès aux activités physiques et sportives des Personnes Vivant avec le VIH en France (PVVIH) cofinancée par Sidaction et par la Région Ile-de-France (2009-2012) ; une étude sur la participation sociale des PVVIH aux activités de loisirs dans une région française (2011-2013) financée dans le cadre des projets permanents de la Caisse nationale de solidarité autonome (CNSA) et de la Direction de la recherche, des études, de l'évaluation et des statistiques (DREES) du Ministère des Affaires sociales et de la Santé.

$\underline{\text { Auteurs : }}$

Sylvain Ferez, Maître de Conférences, Université Montpellier 1

«Santé, éducation, situations de handicap » (EA 4614).

UFR STAPS de Montpellier

700 avenue du Pic Saint-Loup

34090 Montpellier - France

Tél. : 0033 (0)4 11759088

sylvain.ferez@univ-montp1.fr

Estelle Marin-Duval, Doctorante en sociologie, Université Montpellier 3

«Santé, éducation, situations de handicap » (EA 4614)

UFR STAPS de Montpellier

700 avenue du Pic Saint-Loup

34090 Montpellier - France

duvalestelle@gmail.com

Julie Thomas, Post-Doctorante, Université Montpellier 1

«Santé, éducation, situations de handicap » (EA 4614)

UFR STAPS de Montpellier

700 avenue du Pic Saint-Loup

34090 Montpellier - France

juliethomas.professionnel@gmail.com

Stéphane Héas, Maître de Conférences HDR, Université de Rennes 2

«Violences Identités Politiques Sports » (EA 4636).

UFR STAPS - Université Rennes 2

Campus la Harpe - Avenue Charles Tillon - CS 24414 - 35044 RENNES CEDEX

stephane.heas@univ-rennes2.fr

Patrick Fougeyrollas, Ph.D, Professeur associé au département d'anthropologie de l'Université Laval et chercheur au Centre interdisciplinaire de recherche en réadaptation et intégration sociale (CIRRIS).

IRDPQ-IU

525 boul. Wilfrid-Hamel, Québec, Qc G1M 2S8

Tél. : 418-529-9141, poste 6229

Patrick.Fougeyrollas@irdpq.qc.ca 


\section{Résumé :}

Avec le développement des trithérapies et l'allongement de la durée de vie avec le VIH, de nouvelles questions se posent aujourd'hui quant à l'impact du diagnostic, et de la prise en charge à laquelle il confronte. Quand certains parviennent à résister aux injonctions biomédicale à la prise en charge et à préserver les loisirs d'avant le diagnostic, d'autres interrompent ces activités sociales et de loisirs, ou s'engagent dans des dispositifs associatifs où les loisirs deviennent un outil de prise en charge. L'article présente des données recueillies dans deux enquêtes : une sur l'engagement dans les loisirs physiques et sportifs des Personnes Vivant avec le VIH (PVVIH), par questionnaire $(n=619)$, et une sur la participation sociale, par entretien semi-directif $(n=21)$ et par observation participante des dispositifs de loisirs proposés par quatre associations du Languedoc-Roussillon. Elles montrent que les trajectoires de loisirs sont à la fois liées aux situations sanitaires et sociales et au type d'expérience du VIH. Les loisirs ne dérogent pas à l'alternative entre deux logiques, une de préservation d'une vie "normale » et une d'appropriation du statut de malade chronique, qui traversent par ailleurs tous les domaines de l'existence des PVVIH.

\section{Abstract:}

New questions are arising today with the development of highly active antiretroviral therapies (HAART) and a longer life expectancy for people with the HIV, questions concerning the impact of the diagnosis and the medical care proposed. Some people manage to resist biomedical care orders and to preserve the leisure time they engaged in prior to the diagnosis, while others give up these social and leisure time activities or get involved in associations where the activities become part of the planned therapy. The data in the article were collected during two surveys: one about the involvement in sports and physical activities of people living with the HIV (PLWHIV), with a questionnaire $(n=619)$, and one about social involvement, with semi structured interviews $(n=21)$ and by participant observation of the activities proposed by four associations in LanguedocRoussillon. The data show that the choice of spare time activities is linked to the social and health situations of the participants together with the kind of experience of HIV that they have had. Leisure time activities do not go against the alternative between two logics, either to preserve a "normal" life or to appropriate the status of a chronically sick person; furthermore these two logics go through every field of the life of the PLWHIV. 
Une vingtaine d'années après le début de l'épidémie de sida, Mellini et al. (2004) concluaient à la nécessité d'évaluer les effets réels du processus de normalisation sociale du VIH. Soit, au-delà des aspects médicaux, de mieux comprendre cette normalité en action, voire sa réelle banalisation. Mesurer en somme l'impact de la «chronicisation» de la maladie sur les vies des individus concernés et de leurs proches et sur les représentations du VIH/sida. Car, «l'espérance de vie s'étant rallongée, les enjeux du dire mais surtout ceux du taire s'étalent dans le temps et se diversifient (...). Dans un tel scénario, si le but est de poursuivre sa carrière professionnelle, de commencer une relation stable, d'avoir des enfants ou simplement de fréquenter un club de loisirs, la séropositivité n'est peut-être pas tout de suite dévoilée, voire jamais, en fonction des enjeux attachés aux différents contextes de vie » (Mellini et al., 2004 : 163).

Où en sommes-nous une décennie plus tard ? La normalisation décrite a-t-elle favorisé l'accès des personnes vivant avec le VIH (PVVIH) aux loisirs, notamment physiques et sportifs ? Par-delà le poids des représentations, l'enjeu ici est d'étudier l'évolution des expériences de loisir suite au diagnostic, en les resituant dans le cadre des trajectoires sociales et des attentes sanitaires à l'égard des malades chroniques.

\section{1- Entre logique de prise en charge et maintien d'un lien social « normal »}

S'interroger sur l'effet des représentations du VIH/sida sur l'accès aux loisirs implique de questionner le stigmate que constitue toujours cette infection. Le sida, « maladie de la honte », reste associé à une faute dans sa dimension religieuse (Haddad, 2011) ou à un défaut de responsabilité plus spécifiquement reproché aux gays (Jablonski et al., 2010 : 58). Pour la personne diagnostiquée, la honte entraine le retrait plus ou moins radical des relations sociales ordinaires.

En outre, avec la survenue de déficience(s), l'ensemble des formes de participation sociale par le travail, la vie associative, l'engagement politique, etc., mais aussi par l'inscription dans des activités de loisirs plus ou moins codifiées - se trouve affecté (Fougeyrollas, 2010). Le principal obstacle à la participation sociale n'est alors lié aux caractéristiques individuelles que dans la mesure où ces caractéristiques confrontent à des représentations négatives dans les environnements sociaux. La discrétion et/ou le retrait ne visent qu'à réduire - et parfois annuler, lorsqu'ils conduisent à s'auto-exclure - le risque de découverte par autrui d'un trait discréditable (Goffman, 1968). La gestion des informations sur soi, sur son corps, devient un élément fondamental d'un self control censé protéger de la souillure sociale potentielle. Les loisirs collectifs peuvent en ce sens constituer un espace dangereux qu'il s'agit de maîtriser au mieux, quitte à l'éviter.

\section{1-1- Culpabilité et prescription}

Dès le diagnostic prononcé, le VIH opère une contamination du sens (Le Breton, 1998) générant, sinon une rupture biographique (Bury, 1982), tout du moins des perturbations qui menacent la continuité identitaire jusque dans sa mise en récit (Pierret, 2006). L'épreuve du diagnostic produit une reconfiguration symbolique et matérielle qui affecte tout autant le vécu et l'identité de l'individu désormais infecté que l'organisation de son temps quotidien. Le vécu plus ou moins honteux et l'expérience de la fragilité qui l'accompagne s'accommodent ainsi d'un temps réorganisé autour de la prise en charge et des rythmes qu'elle impose (Langlois, 2006). Le loisir a-til encore une place dans cette nouvelle organisation temporelle?

En pleine phase de questionnement moral et de reconfiguration identitaire, au moment même où il est souvent conduit à interrompre sa sexualité (Duval et al., 2012), l'individu éprouve-t-il le besoin d'avoir des plaisirs et/ou des loisirs ? Se sent-il seulement autorisé à en avoir ? Car le plaisir n'a pas toujours sa place, comme le rappelle une PVVIH interrogée par Stephens : «le plaisir n'entre jamais dans l'équation. La guerre du sida se mène sur mon corps $»^{1}$. Les PVVIH ne seraient-

\footnotetext{
${ }^{1}$ Cité par Hurley (2010 : 126).
} 
elles autorisées à jouir qu'à la condition de se conformer aux prescriptions médicales ? Le fond de culpabilité qui les habite n'est-il pas mis au service de l'entreprise de responsabilisation de soi commandée par les environnements médicosociaux de prise en charge qui leur demandent (depuis l'arrivée des trithérapies en 1996) d'entrer dans une carrière de malade chronique, en observant scrupuleusement son traitement, en mettant en place une hygiène de vie irréprochable et en remodelant ses habitudes de vie autour du VIH (Pierret, 1997 ; Corbin et Strauss, 1991) ?

$\mathrm{La}$ «chronicisation » de la maladie exprime davantage, selon nous, un changement des attentes sociales à l'égard des PVVIH imposé par les systèmes de prise en charge, qu'une évolution strictement liée à la découverte de traitements repoussant la fatalité objective de la mort (Ferez et Thomas, 2012). Sa logique ne trouve-t-elle pas son aboutissement dans un fantasme de «gouvernement des corps » (Memmi et Fassin, 2004) qui peut affecter la sphère des loisirs ? Si depuis la seconde moitié du $20^{\mathrm{e}}$ siècle, les loisirs s'inscrivent de plus en plus dans la revendication d'un temps pour soi (en faisant un mode d'expression, de re-création et d'invention de soi), une visée prescriptive les hante toujours.

Cette visée est liée, entre autres, au partage introduit dans la seconde moitié du $19^{\mathrm{e}}$ siècle par les élites occidentales entre un loisir rationnel et légitime, susceptible de moraliser le peuple, et des formes peu respectables de divertissement : «Longtemps, la recherche du bon loisir, l'exaltation de ses vertus préventives et thérapeutiques se sont imposées »(Corbin, 1995: 13). Loin d'avoir totalement disparue, la prescription de loisirs prend aujourd'hui de nouvelles formes, comme en atteste le glissement d'un usage socioéducatif vers un usage lié à la problématique sécuritaire (Saez, 2002). Les systèmes de prise en charge suite au diagnostic de VIH conduisent, depuis sa redéfinition comme maladie chronique, à l'exercice plus ou moins marqué de formes de contrôle des loisirs par de véritables entrepreneurs de morale (Becker, 1963).

\section{1-2- Loisirs, normalité et intégration sociale}

Dans quelle mesure et jusqu'à quel point le désir des personnes diagnostiquées d'assurer une continuité du vécu et de l'expérience, même si elle est fragile et sous tension (Pierret, 2006 ; Langlois, 2006), conduit-il à maintenir les activités antérieures, comme si de rien n'était, et à résister à cette prescription de «bons loisirs »? Les loisirs sont en effet devenus à la fois un vecteur et un marqueur de normalité et d'intégration sociale en France où ils deviennent un élément central de la culture dès les années 1960 (Dumazedier, 1962).

Pour Yonnet, les loisirs relèvent d'ailleurs aujourd'hui d'une fonction de substitution à la religion, comme manière de re-lier. Loin de renvoyer à des pratiques futiles, leur substance même est le lien social. Dans ce cadre, la demande de loisirs se constitue à la fois comme une demande de sociabilité et comme une demande de séparation des temps d'activité, notamment par la distinction du temps de travail et du temps libéré à l'égard de ce dernier : "Ce qui identifie la normalité sociale ou, si l'on veut, le critère par lequel les agents sociaux jugent de leur degré de normalité ou d'intégration sociale, c'est la possibilité pour eux de traverser alternativement, dans la journée, la semaine ou l'année, des temps d'obligations et de contraintes, et des tranches horaires libératoires de ceux-ci » (Yonnet, $1999:$ 33).

Le diagnostic de VIH, en désorganisant à la fois les bases de la sociabilité et l'organisation temporelle du quotidien, ne vient-il pas saper le sens même du loisir ? La difficulté à maintenir une structuration du temps alternant le travail et le loisir ne menace-t-elle pas, comme lorsque le chômage s'éternise, de conduire sur la voie de la marginalisation?

\section{1-3- Quels loisirs pour les exclus?}

L'épidémie de sida émerge dans les années 1980, au moment où la crise du travail et les doutes sur la «civilisation du loisir» s'accroissent (Dumazedier, 1988). L'essor des loisirs, publics ou privés, est alors placé sous le signe de la démocratisation et associé à une représentation humaniste et intégratrice. Dans ce contexte, la situation des exclus, chômeurs ou marginaux interroge : ceux 
qui ne travaillent pas peuvent-ils avoir des loisirs ? Ceux qui sont insolvables peuvent-ils accéder aux loisirs marchands ? Avoir un loisir ne devient-il pas « un moyen de gagner du prestige à leurs propres yeux s'ils ne peuvent en avoir par le travail » (Saez, 2002:12) ?

Si le diagnostic du VIH soumet plus ou moins à la spirale de la précarisation, il confronte dans tous les cas à une réorganisation du temps et à la crainte de l'exclusion. Les loisirs conservent-ils une place dans l'existence des PVVIH ? Sont-ils des agents d'intégration et de normalité, qui préservent un lien social menacé - a fortiori à une époque marquée par la dilution et la fragilisation de ce lien, que les individus cherchent à tout prix à retisser (Bouvier, 2005) ? S'inscrivent-ils par exemple davantage dans une «moralité du plaisir» et dans le registre de «bonnes pratiques » prescrites par les environnements de prise en charge ?

La sociologie du VIH s'est surtout constituée dans le cadre d'une socio-anthropologie de la maladie et de la médecine (Adam et Herzlich, 1994) centrée sur les trajectoires de soins et la gestion de l'infection dans les environnements familiaux et professionnels. La question des loisirs demeure marginale, exceptée pour les « loisirs festifs » pouvant impliquer des usages à risque en matière de sexualité ou de consommation de drogues, notamment chez les gays (Jablonski et al., 2010). En interrogeant la redéfinition de l'expérience des loisirs des PVVIH suite au diagnostic, il s'agit ici de voir dans quelle mesure et jusqu'à quel point ils représentent pour eux une occasion de prise de pouvoir culturel et d'émergence de nouvelles sociabilités et dynamiques sociales, ou bien figurent, au contraire, l'emprise des systèmes de prise en charge sur leur nouvelle vie de malades chroniques.

\section{1-4- Quels déterminants de l'évolution de la sociabilité de loisir chez les PVVIH?}

Les résultats présentés s'appuient sur des données collectées dans le cadre de deux enquêtes : une sur l'accès des PVVIH aux activités physiques et sportives (APS) en France (Ferez \& Thomas, 2012), l'autre sur la participation sociale des PVVIH aux activités de loisirs ${ }^{2}$. L'enjeu ne sera pas ici d'évaluer l'évolution du temps consacré aux loisirs - aux activités sportives, culturelles, etc. - à la suite du diagnostic, en recourant aux méthodes d'analyse des emplois du temps (Pronovost, 2002). Il ne s'agira pas non plus d'essayer de qualifier le loisir en lui-même, et pour lui-même, à partir de ses fonctions, ou de caractéristiques qui lui seraient propres.

Nous interrogeons les usages du «temps libre » au regard de la manière dont ils peuvent (on non) être vécus sous le mode du loisir (Teboul, 2004). Le loisir sera ainsi appréhendé comme un temps libéré des contraintes professionnelles - lorsque l'activité a été maintenue après le diagnostic - (Yonnet, 1999), mais aussi de celles liées à la prise en charge du VIH et à la carrière de «malade chronique» (plus ou moins docilement embrassée). Outre cette inscription dans l'organisation sociale du temps - et la prise en compte de ses effets sur le vécu -, le loisir sera par ailleurs conçu au regard de son rapport à la sociabilité : il ne sera pas question, comme dans les enquêtes réalisées par l'INSEE, de distinguer le temps consacré à l'un et à l'autre.

$\mathrm{Au}$ contraire, le traitement portera sur une question de l'enquête «Sport et VIH » $(n=619)$ : «Depuis la découverte de votre séropositivité, comment ont évolué vos activités sociales et de loisirs ? $\left(\right.$ cf. encadré $\mathrm{n}^{\circ} 1$ ). La formulation «activités sociales et de loisirs » visaient délibérément à appréhender le loisir dans le cadre d'une recherche de sociabilité, plutôt qu'au regard de l'identification d'activités socialement codifiées. Enfin, parmi les déterminants de l'évolution de l'expérience des loisirs pris en compte, outre l'attention aux situations sanitaires et sociales des individus, un regard particulier sera porté sur les dispositifs de loisirs spécifiquement adressées aux PVVIH. L'analyse de cette offre de pratique s'appuiera sur des données recueillies lors d'une étude régionale française (cf. encadré $\mathrm{n}^{\circ} 2$ ).

Dans le cas qui nous intéresse, on ne peut entièrement rendre compte du vécu des loisirs des PVVIH sans resituer leur expérience, non seulement dans leurs trajectoires sanitaires et sociales depuis le diagnostic, mais aussi dans le cadre d'attentes sociales à leurs égards qui se manifestent notamment dans l'offre de loisirs spécifiques qui leur est proposée.

\footnotetext{
${ }^{2}$ La première a été cofinancée par Sidaction et la région Ile-de-France, la seconde par la Drees/MiRe-CNSA.
} 


\section{Encadré $\mathrm{n}^{\circ} 1$ : L'accès aux Activités Physiques et Sportives (APS) des PVVIH}

Cette enquête nationale menée grâce au soutien financier de Sidaction et de la région Île-deFrance (2009-2011) a permis de recueillir cinquante entretiens non-directifs et 619 questionnaires. Une première version du questionnaire a été créée en lien avec des représentants d'associations VIH et des PVVIH, avant d'être testée auprès de 15 personnes. Sa version finale renseigne des indicateurs sur la gestion de l'information sur sa séropositivité, le rapport à la prise en charge, les capacités et incapacités, le rapport au corps, les trajectoires sportives et de loisirs, la situation socioprofessionnelle et sociodémographique.

L'administration a été réalisée en face à face ou par téléphone (30 minutes à 2 heures suivant la complexité des trajectoires et l'état de santé des personnes). L'échantillonnage a respecté un principe de diversification maximale des profils de répondants au regard de la pratique des APS, mais aussi des modes de contamination, de l'ancienneté du diagnostic, des modes de prise en charge du VIH, des situations sanitaires, professionnelles et sociales. Le traitement des données, effectué sous Modalisa 6.0, visait à identifier les effets communs du diagnostic sur la problématique de l'accès aux APS, et l'hétérogénéité des ressources et stratégies pour gérer cette problématique.

Encadré $\mathrm{n}^{\circ} 2$ : Etude de la participation sociale des PVVIH aux activités de loisirs

Cette étude s'appuie sur l'observation participante de dispositifs de loisirs mis en place par les associations VIH d'une région française, complétée par 4 entretiens avec des responsables de ces structures. Ces données ont par ailleurs été enrichies par 9 entretiens non directifs et 13 entretiens semi-directifs ${ }^{3}$ avec des PVVIH de profils diversifiés (en fonction de leur investissement ou non dans des associations, de leur sexe, de leur orientation sexuelle, de leur engagement professionnel, du mode de contamination et de l'ancienneté du diagnostic). Le choix des activités observées a été négocié avec les responsables associatifs. Ont été inclues toutes activités pouvant être définies, selon l'avis des dirigeants, comme une activité de «loisir»par les participants. L'enquêteur a participé aux activités de 4 associations (deux « communautaires ${ }^{4} »$ et deux $«$ de services $\left.{ }^{5} »\right)^{6}$. La participation a été régulière et continue durant 4 mois, de mars à juin 2012. Ont ainsi été observés :

1) Parmi les associations de service: des ateliers visant l'amélioration de la qualité de vie des bénéficiaires (cuisine, gymnastique d'entretien, natation, aquagym), des sorties culturelles, des ateliers psychothérapeutiques «ludiques », un repas de fin d'année ;

2) Parmi les associations communautaires : des randonnées, des « réunions d'informations sociales et conviviales », des « convivialités »;

3) Des activités en marge des associations de service (organisées pendant des activités associatives, mais ayant lieu dans un espace et à un moment autre) : des repas suivant les ateliers associatifs, ainsi qu'un week-end organisé entre les PVVIH.

Les notes d'observation ont systématiquement été prises à l'issue des activités. La grille d'observation accordait une attention spécifique aux interactions (verbales et non verbales) entre participants en fonction de leurs rôles (dirigeants, professionnels, PVVIH, bénévoles). Les données

\footnotetext{
${ }^{3}$ La grille d'entretien a été construite à partir d'une analyse exploratoire des entretiens non-directifs.

${ }^{4}$ Terme émique utilisé par un dirigeant pour désigner les associations dont les membres (salariés, bénévoles, adhérents) sont uniquement des PVVIH ou des PVVHC ou leurs proches (même si ceux-ci ne participent en réalité jamais).

5 Terme émique utilisé par une ancienne «usagère » des deux associations pour désigner des organisations destinées aux PVVIH (ou aux malades chroniques) qui sont dirigées par des professionnels, a priori séronégatifs.

${ }^{6}$ Ces associations datent toutes des années 2000. Alors que les associations communautaires sont principalement constituées de PVVIH hétérosexuelles (hommes et femmes), les associations de services accueillent davantage des femmes hétérosexuelles et des hommes homosexuels.
} 
documentaires sur les différentes activités (sites Internet et documents de présentation) et les entretiens avec les responsables (données sur 1'histoire des associations) ont été intégrées à l'analyse, conduite à partir de la rédaction de fiches synthétiques par activité, puis de fiches thématiques transversales.

\section{2- Perturbation biographique et évolution des loisirs : des trajectoires diverses...}

\section{2-1 L'effet des situations sanitaires et sociales sur les loisirs}

Une seule des questions de l'étude «Sport et VIH » portait directement sur l'évolution de ce que l'on a nommé dans le questionnaire les «activités sociales et de loisir » (ASL) depuis le diagnostic, proposant cinq possibilités : l'absence de changement du poids de ces activités, une augmentation de leur importance, une fluctuation, une diminution ou un arrêt. Des analyses bivariées ont permis de dégager des régularités dans les caractéristiques sanitaires et sociales des répondants. Les profils bien distincts des interrogés selon la modalité choisie ont permis de former cinq groupes sur la base des réponses à cette question. Seules sont ici présentées les variables pour lesquelles les croisements avec la variable «évolution des ASL » sont significatifs au test du Chi2 de Pearson pour les variables nominales, et suite à une analyse de la variance au F-test sur variable numérique pour les variables d'échelle ; la fiabilité des tests a été vérifiée selon les propositions de Lemelin (2004). Pour ces variables, les modalités présentant une attraction significative - mesurées par le Pourcentage de l'Ecart Maximum (Cibois, 1993) - avec les modalités de la variable dépendante sont les seules exposées.

Des relations fortes apparaissent entre d'une part la situation sanitaire des individus, leur trajectoire médicale - plus ou moins longue, marquée par des maladies et affectée par les traitements -, l'évolution de leur poids, leur niveau d'incapacité, leur rapport au traitement et leur connaissance du/de « leur » VIH ; et d'autre part l'évolution de leurs ASL A $^{7}$. On note des oppositions structurantes entre chacun des groupes. La déclaration de l'évolution des ASL, si elle n'est sans relation avec des situations sanitaires spécifiques, entretient aussi des liens avec les situations sociales des répondants. Le tableau 1 regroupe les résultats qui spécifient les profils sociaux des répondants de chaque groupe (lorsqu'elle n'est pas précisée, la p-value du test est inférieure à $\mathrm{p}=0.01)$.

\begin{tabular}{|c|c|c|c|c|c|}
\cline { 2 - 5 } \multicolumn{1}{c|}{} & $\begin{array}{c}\text { ASL stables } \\
\mathbf{n = 2 4 5}(\mathbf{G 1})\end{array}$ & $\begin{array}{c}\text { ASL accrues } \\
\mathbf{n = 8 0}(\mathbf{G 2})\end{array}$ & $\begin{array}{c}\text { ASL fluctuantes } \\
\mathbf{n = 8 7}(\mathbf{G 3})\end{array}$ & $\begin{array}{c}\text { ASL réduites } \\
\mathbf{n = 1 2 6}(\mathbf{G 4})\end{array}$ & $\begin{array}{c}\text { ASL } \\
\text { interrompues } \\
\mathbf{n = 3 8}(\mathbf{G 5})\end{array}$ \\
\hline Sexe et orientation sexuelle & $\begin{array}{c}\text { Homme } \\
\text { homosexuel }\end{array}$ & $\begin{array}{c}\text { Homme } \\
\text { homosexuel }\end{array}$ & $\begin{array}{c}\text { Femme } \\
\text { hétérosexuelle }\end{array}$ & $\begin{array}{c}\text { Femme } \\
\text { (sous-représentation } \\
\text { des gays) }\end{array}$ & $\begin{array}{c}\text { hétérosexuelle ou } \\
\text { «tre } »(=a b s e n c e \\
\text { de sexualité) }\end{array}$ \\
\hline $\begin{array}{c}\text { Migrations (lieu de naissance et } \\
\text { nationalité) }\end{array}$ & $\begin{array}{c}\text { Né en France } \\
\text { métropolitaine }\end{array}$ & $\begin{array}{c}\text { Né en Afrique du } \\
\text { Nord }\end{array}$ & $\begin{array}{c}\text { Non significatif } \\
(N S)\end{array}$ & $\begin{array}{c}\text { Né dans les } \\
\text { départements ou } \\
\text { territoires français } \\
\text { d'Outre-Mer }\end{array}$ & $\begin{array}{c}\text { Né en Afrique } \\
\text { noire ; nationalité } \\
\text { non française }\end{array}$ \\
\hline
\end{tabular}

\footnotetext{
${ }^{7}$ Plus précisément, les liens statistiques (dont la significativité des tests est supérieure à 99\%) permettant de dégager des profils d'individus tout à fait différenciés ont été établis à partir d'une série de variables liées à la trajectoire médicale : date du diagnostic; maladie/s grave/s depuis le diagnostic; pathologie/s grave/s depuis le diagnostic oui/non; pathologie/s grave/s depuis le diagnostic ; mode de contamination; médicaments psychotropes consommés ; évolution du poids ; déclaration ou non de déficiences depuis le diagnostic et taux d'incapacité (si la reconnaissance a été obtenue) ; observance thérapeutique déclarée ; connaissance de son taux de CD4 et de sa charge virale. Ont été ajoutées à cette série de variables: 1) celles liées à la connaissance du VIH en raison de la fréquence de la recherche d'informations le concernant ; 2) celles liées au recours à d'autres types de prise en charge que les médicaments ; 3) celles liées à la déclaration ou non d'effets secondaires des traitements ; 4) celles en rapport avec l'évolution des ASL avec une probabilité de plus de $95 \%$.
} 


\begin{tabular}{|c|c|c|c|c|c|}
\hline $\begin{array}{l}\text { Trajectoires professionnelles } \\
\text { (situation professionnelle actuelle, } \\
\text { niveau de diplôme, évolution de } \\
\text { l'activité professionnelle) }\end{array}$ & $\begin{array}{c}\text { En activité } \\
\text { professionnelle, } \\
4 \text { années } \\
\text { d'études } \\
\text { validées après le } \\
\text { baccalauréat } \\
\text { (BAC+4) et plus }\end{array}$ & $\begin{array}{l}\text { En invalidité, } \\
\text { BAC }+2 / 3, \\
\text { investissement } \\
\text { professionnel } \\
\text { augmenté }\end{array}$ & $\begin{array}{l}\text { En invalidité, } \\
\text { investissement } \\
\text { profession } \\
\text { interrompu }\end{array}$ & $\begin{array}{c}\text { Sans activité ou en } \\
\text { arrêt longue } \\
\text { maladie, études } \\
\text { primaires ou } \\
\text { secondaires } \\
\text { investissement } \\
\text { professionnel } \\
\text { interrompu ou } \\
\text { réduit } \\
\end{array}$ & $\begin{array}{l}\text { En invalidité, pas } \\
\text { de diplôme }\end{array}$ \\
\hline Lieu de résidence $(\mathrm{p}<0.05)$ & $N S$ & $N S$ & $\begin{array}{c}\text { Grande ville en } \\
\text { région }\end{array}$ & $N S$ & Ile-de-France \\
\hline $\begin{array}{l}\text { Revenus, bénéfice d'aides et } \\
\text { déplacements }\end{array}$ & $\begin{array}{l}\text { Revenus très } \\
\text { élevés, } \\
\text { aucune aide }\end{array}$ & $N S$ & $\begin{array}{l}\text { Aides liées à son } \\
\text { état sanitaire } \\
\text { (Allocation } \\
\text { d'Adulte } \\
\text { Handicapé, mi- } \\
\text { temps } \\
\text { thérapeutique, } \\
\text { pension } \\
\text { d'invalidité) }\end{array}$ & $\begin{array}{l}\text { Revenus faibles, } \\
\text { minimas sociaux, } \\
\text { difficultés pour se } \\
\text { déplacer }\end{array}$ & $\begin{array}{c}\text { Revenus très } \\
\text { faibles, aides liées } \\
\text { à son état } \\
\text { sanitaire } \\
\text { (sous-représentation } \\
\text { de l'usage d'une } \\
\text { voiture) }\end{array}$ \\
\hline $\begin{array}{l}\text { Engagement associatif et structure } \\
\text { d'orientation des interrogés }\end{array}$ & $\begin{array}{l}\text { Aucun } \\
\text { engagement } \\
\text { associatif, } \\
\text { orienté via } \\
\text { l'hôpital }\end{array}$ & $\begin{array}{c}\text { Dans des } \\
\text { associations avec } \\
\text { ou sans lien avec } \\
\text { le VIH, orienté } \\
\text { via une } \\
\text { association VIH }\end{array}$ & $\begin{array}{c}\text { Orienté via } \\
\text { Internet, forums, } \\
\text { etc. (hors } \\
\text { association ou } \\
\text { hôpital) }\end{array}$ & $\begin{array}{l}\text { Orienté via une } \\
\text { association VIH }\end{array}$ & $\begin{array}{c}\text { Dans une } \\
\text { association VIH, } \\
\text { orienté vers } \\
\text { l'enquête par } \\
\text { celle-ci }\end{array}$ \\
\hline $\begin{array}{l}\text { Orientation politique ; foi } \\
\text { religieuse }(\mathrm{p}<0.05)\end{array}$ & $N S$ & $N S$ & $\begin{array}{l}\text { De gauche, non } \\
\text { croyant }\end{array}$ & $\begin{array}{c}\text { Refus de dire son } \\
\text { orientation } \\
\text { politique, croyant } \\
\text { pratiquant }\end{array}$ & $\begin{array}{l}\text { Croyant (sous- } \\
\text { représentation des } \\
\text { votants à gauche) }\end{array}$ \\
\hline $\begin{array}{l}\text { Reconnaissance administrative du } \\
\text { handicap (Reconnaissance de Qualité } \\
\text { de Travailleur Handicapé ; demande de } \\
\text { reconnaissance du taux d'incapacité) }\end{array}$ & $\begin{array}{l}\text { Aucune } \\
\text { demande }\end{array}$ & $N S$ & $\begin{array}{l}\text { Reconnaissance } \\
\text { de Qualité de } \\
\text { Travailleur } \\
\text { Handicapé }\end{array}$ & $\begin{array}{c}\text { Taux d'incapacité } \\
\text { demandé et } \\
\text { reconnu }\end{array}$ & $\begin{array}{c}\text { Reconnaissance } \\
\text { de Qualité de } \\
\text { Travailleur } \\
\text { Handicapé et taux } \\
\text { d'incapacité } \\
\text { demandés et } \\
\text { reconnus } \\
\end{array}$ \\
\hline $\begin{array}{c}\text { Couple (type de ménage } \mathrm{p}<0.05 \text {; forme } \\
\text { légale de couple } \mathrm{p}=0.05 \text { ) }\end{array}$ & $\begin{array}{c}\text { En couple, au } \\
\text { sein d'un pacte } \\
\text { civil de } \\
\text { solidarité (forme } \\
\text { de concubinage } \\
\text { acté par la loi) } \\
\text { ou marié }\end{array}$ & $N S$ & Veuf/veuve & $N S$ & $N S$ \\
\hline
\end{tabular}

Tableau 1 : Situations sociales fréquentes selon l'évolution des ASL depuis le diagnostic

Les ressources sociales, économiques et culturelles semblent bien peser sur l'évolution des ASL suite au diagnostic. Moins affectés corporellement par des maladies, des déficiences ou des effets indésirables des traitements, les personnes dont les ASL sont restées stables $(G 1,42 \%)-$ souvent diagnostiqués après 2000 et rapidement mises sous traitement - sont également beaucoup moins affectées socialement. Ce sont en effet celles qui continuent le plus souvent à travailler, avoir des revenus importants (grâce à leur niveau d'études plus élevés) et à vivre en couple stable. Ces ressources - culturelles, financières, sociales - et/ou leur contamination récente les amènent à minorer l'attention qu'elles accordent à leur santé. Bien qu'elles soient d'une observance thérapeutique sans faille, elles ne s'informent jamais sur le VIH et n'ont pas recours à d'autres types de prise en charge que les médicaments. Ces personnes affirment massivement, dès qu'elles en ont l'occasion, être restées « comme avant».

G2 (14\% des interrogés) est, comme G1, composé en majorité d'hommes gays contaminés lors d'une relation homosexuelle. Mais il rassemble surtout des répondants diagnostiqués avant 1986,

\footnotetext{
${ }^{8}$ Les études primaires sont en moyenne achevées par les élèves français dans leur onzième année. Le niveau «études primaires » signifie que l'interrogé.e n'a pas suivi de cours dans l'enseignement secondaire français (collège ou lycée), le niveau «études secondaires » que l'interrogé.e n'a pas été jusqu'au baccalauréat ou jusqu'à un diplôme technique comme le Certificat d'Aptitude Professionnelle (CAP) ou le Brevet d'Etudes Professionnelles (BEP).
} 
des «survivants » de l'épidémie qui ont connu les pathologies graves (tels les sarcomes de Kaposi et les pneumocystoses) qui marquaient les parcours morbides des premiers malades. D'ailleurs, ils sont parfois en invalidité, même s'ils ont tendance à augmenter aussi leur activité professionnelle lorsqu'ils le peuvent. En fait, depuis que l'épée de Damoclès du sida s'est éloignée, surveillant activement leurs paramètres biologiques, ils prennent massivement en charge leur santé, surtout via les ASL, notamment la pratique physique. On peut remarquer par ailleurs qu'ils annoncent bien plus que les autres répondants pratiquer une activité physique pour contrer les effets des traitements (et sur le conseil d'un médecin), et ressentir une forte contrainte à la pratique physique, souvent perçue par ces répondants comme un moyen de prendre en charge leur VIH. Ces répondants prétendent d'ailleurs fréquemment qu'ils n'aiment l'activité physique que depuis leur séropositivité. Les personnes recrutées par le biais des associations VIH sont surreprésentées dans ce groupe.

Le groupe aux ASL fluctuants $(G 3,15 \%)$ est davantage constitué de femmes hétérosexuelles et de personnes contaminées lors d'injection de drogues par intraveineuses, veuves, dont le diagnostic est relativement ancien. On retrouve ici souvent des personnes avec des trajectoires professionnelles fluctuantes, liées à des incapacités qui ont fait l'objet d'une demande de reconnaissance officielle. Ces PVVIH déclarent fréquemment l'expérience de maladies graves et/ou de déficiences, un poids fluctuant, et se plaignent davantage d'effets secondaires des traitements. Ces divers problèmes ont alimenté le souci d'être expertes de leur VIH et une tendance à la surveillance permanente de soi : se peser, connaitre ses paramètres biologiques, s'informer plusieurs fois par semaine sur le VIH, se tourner vers des médecines parallèles, consulter un psychothérapeute, et, comme $G 2$, pratiquer une activité physique pour contrer les effets des traitements.

Les personnes qui déclarent une réduction de leurs ASL $(G 4,22 \%)$ ont été diagnostiquées assez récemment, généralement après l'arrivée des premières trithérapies, et plus souvent que les autres à la suite d'un accident d'exposition du sang. Le vécu négatif du VIH (que révèle leur tendance à la consommation d'antidépresseurs et de somnifères) et/ou l'expérience de déficiences souvent reconnues comme incapacitantes à plus de $80 \%$ - affectent fortement leurs parcours professionnels. Malgré une fréquente reconnaissance de handicap, leurs ressources proviennent plus souvent des aides «sociales» que des aides sanitaires (comparés aux autres répondants). Les individus de ce groupe sont davantage caractérisés par une interruption précoce des études, un assez faible niveau de revenus, l'absence d'activité professionnelle. Ils ont fréquemment été orientés vers l'enquête par une association de lutte contre le VIH.

Enfin, parmi ceux qui affirment avoir cessé toute ASL depuis leur diagnostic $(G 5,7 \%)$, nombreux font état de déficiences. Dans ce groupe, les demandes de reconnaissance du handicap pour le milieu professionnel (RQTH et/ou invalidité) ou pour bénéficier d'aides sanitaires (AAH) sont surreprésentées, et les répondants les ont souvent obtenues. Pour autant, ils semblent peu connaitre et prendre en charge leur VIH (oubli assez fréquent du traitement, méconnaissance de leurs paramètres biologiques et du mode de contamination). Surreprésentés parmi ceux dont le poids a augmenté ou fluctué depuis le diagnostic, ils fréquentent plus volontiers les associations de lutte contre le VIH, qui sont massivement à l'origine de leur orientation vers les enquêteurs. Les répondant de $G 5$ disposent enfin de ressources économiques, culturelles et sociales très spécifiques, puisque nous sommes ici surtout en présence de migrants (souvent des femmes) sans diplôme et à très faibles revenus.

\section{2-2- L'effet de l'investissement dans les associations de PVVIH sur les loisirs}

On peut s'interroger sur le fait que les trois groupes fréquentant davantage les associations VIH $(G 2, G 4, G 5)$ se caractérisent soit par la déclaration d'un accroissement de leurs ASL depuis le diagnostic, soit au contraire par l'affirmation de leur réduction plus ou moins drastique. A l'opposé, les publics non investis dans les associations de lutte contre le VIH déclarent plus volontiers des ASL stables ou fluctuantes. Comment expliquer la dualité du recrutement des associations au regard de cette dimension? Est-elle liée à des publics associatifs ayant un rapport foncièrement différent au loisir et/ou à une dualité de l'offre associative d'ASL ? 
Les quatre associations étudiées ont toutes défini leurs objectifs comme reliés à la prise en charge du VIH, au travers notamment d'activités physiques et sportives, culturelles, artistiques et ludiques. Les deux associations de services visent d'abord une amélioration «la qualité de vie ${ }^{9}$, de l' « accompagnement psychologique, corporel et/ou social » et de la «prise en charge » du VIH. Les associations «communautaires » visent davantage à rendre les PVVIH actrices de leur vie, par le biais d'activités physiques, par le partage de compétences et d'expertises grâce à la " convivialité », ou par la mise à disposition d'informations : "Mieux informés, mieux soignés ». Il convient d'observer que les associations de services ont initialement été mises en place en lien étroit avec le Service des Maladies Infectieuses et Tropicales d'un hôpital et des services sociaux : ce sont d'ailleurs ces services qui leur orientent les « bénéficiaires » et « usagers ${ }^{10}$.

L'offre de loisirs est donc fortement structurée autour d'une volonté de prise en charge à la fois physique (lutter contre les effets de l'infection et les éventuels effets secondaires des traitements), psychologique et social (lutter contre l'isolement afin de favoriser la santé psychologique). Les questions de plaisir, d'occupation du temps libre ou d'épanouissement personnel ne sont pas des objectifs affichés. Ce choix correspond à l'anticipation des attentes des financeurs, avec une certitude : les loisirs ne seraient légitimes, et donc susceptibles de recevoir un financement, que s'ils sont bénéfiques en termes de santé. Le poids des politiques de santé publiques s'esquissent donc, en arrière-plan. Ainsi, s'engager dans une association de prise en charge du VIH oblige à adhérer, plus ou moins fortement et volontiers, à ces objectifs de prise en charge de sa situation physique, sociale et psychologique par le biais, entre autres, d'ASL.

Si ces associations veulent faciliter l'accès aux pratiques sportives ou culturelles en les adaptant à la situation physique et sociale des PVVIH, ne favorisent-elles pas avant tout les objectifs thérapeutiques ? Comment les PVVIH s'approprient-elles les dispositifs proposés en vue d'accomplir ces missions ? Des formes de résistances ne se mettent-elles pas en place ?

\section{3- Conserver des loisirs « normaux » ou devoir les repenser...}

Les loisirs proposés dans les associations VIH semblent à la fois «adaptés » et « contraints », même si la contrainte ne s'apparente ici ni à une nécessité clairement affichée, ni à la prescription d'une autorité établie. Elle relève davantage de l'incorporation quotidienne d'une obligation sans cesse négociée en pratique. Mais l'idée d'une telle contrainte aux loisirs ne constitue-t-elle pas, de fait, ce qui conduit la plupart des PVVIH à rester à l'écart des associations, considérant leur fréquentation comme le signe d'une bascule entérinant la sortie d'une normalité âprement revendiquée ? Les loisirs «normaux » deviennent alors une manière de refuser l'entrée dans une carrière de malade chronique, ou la réduction de son existence à cette dernière.

\section{3-1- Les loisirs comme signe du maintien dans une «normalité » menacée}

Ceux qui déclarent que les ASL ont conservé la même importance après le diagnostic $(G l)$ déclarent fréquemment que le diagnostic n'a pas eu d'impact sur leur existence ou que le VIH les oblige simplement à certains aménagements. Ils sont les seuls qui affirment volontiers que le VIH n'a pas transformé leur regard sur leur corps. Ils rejettent d'ailleurs massivement toutes les propositions (corps déformé, pas séduisant, ou mieux qu'avant) quant aux éventuelles modifications de ce dernier : les scores moyens sont systématiquement les moins élevés de la population. Ils sont particulièrement nombreux à penser que le VIH n'est pas du tout visible sur leur corps ${ }^{11}$. Logiquement, ils sont surreprésentés parmi ceux qui déclarent le meilleur niveau d'acceptation de

\footnotetext{
${ }^{9}$ Un terme à connotation fortement médicalisée.

${ }^{10}$ Ces termes, utilisés par les professionnels de ces structures, ne sont pas toujours appréciés par les individus désignés.

${ }^{11}$ Score moyen de 1.6/5, et surreprésentation de la modalité « il n'est pas du tout visible » (1/5).
} 
leur séropositivité au VIH (affirmant très bien l'accepter) ${ }^{12}$, et parmi ceux qui affirment avoir « rarement » ou «pas du tout » pensé au VIH lors de la semaine écoulée.

Cela n'empêche toutefois pas l'émergence d'une certaine appréhension à vieillir plus vite avec le VIH, puisqu'ils sont surreprésentés chez ceux qui optent pour le choix médian de $3 / 5$. Pour autant, le score moyen de ce groupe pour cette variable (appréhension à vieillir avec le VIH) est le plus bas de la population (les autres groupes déclarent une appréhension plus forte encore). Cette crainte ne semble d'ailleurs pas entraîner de bouleversement de leur attention au corps, puisqu'ils déclarent massivement se regarder dans le miroir et se peser autant qu'avant ${ }^{13}$. Parallèlement, ils affichent également une stabilité dans leur goût pour l'APS.

En outre, les individus de $G 1$ se caractérisent par la tendance plus marquée que le reste de la population à n'avoir informé sur leur statut sérologique ni leurs collègues, ni leur mère et leurs frères et sœurs, et à n'en avoir parlé qu'avec un ou deux amis. Plus nombreux que la moyenne à n'avoir parlé de leur séropositivité qu'à une ou deux personnes, ils sont surreprésentés parmi ceux qui déclarent ne côtoyer aucune autre PVVIH dans la semaine. Puisque le VIH ne change rien à leur vie, pourquoi en informeraient-ils leurs proches ? Pourquoi fréquenteraient-ils d'autres personnes infectées ? Le cas de Roland est exemplaire de ce type d'expérience (cf. encadré $n^{\circ} 3$ ).

\section{$\underline{\text { Encadré } n^{\circ} 3}$ : Une trajectoire de loisirs presque «normale »}

Roland, homosexuel, 34 ans, diagnostiqué depuis 10 ans au moment de l'entretien, affirme que sa trajectoire de loisirs n'a pas subi d'impact important, malgré les perturbations du diagnostic (« un temps d'adaptation », une «phase à vide »). Si la fréquence de ses activités physiques est plus importante aujourd'hui, ce n'est pas lié, selon lui, au diagnostic: il avait arrêté une pratique de judo de haut niveau avant ce dernier, et a débuté un autre sport de combat quelques temps après. La différence résiderait finalement moins dans les activités que dans l'invisibilité de sa séropositivité : «J'avais les mêmes activités, sauf... sauf que personne ne savait pour moi en fait ! ». Il précise que s'il n'a «jamais eu de restriction par rapport à ça », c'est aussi parce qu'il a peu de symptômes et que sa maladie «se passe bien ». Les seules personnes au courant sont sa famille proche, son copain de l'époque et deux ou trois amis. Aujourd'hui, il pratique la boxe, un sport auquel est pourtant généralement associée une forte crainte de contaminer. Suite à son diagnostic, Roland a cherché à s'adresser à une association de PVVIH, parce qu'il n'avait «personne à qui en parler » et voulait rencontrer des gens « comme lui ». La crainte de la visibilité l'a toutefois conduit à faire machine arrière : «J'avais peur que ça se répète ». Depuis, il n'est plus retourné dans une association.

Comme ceux de $G 1$, les répondants du groupe $G 2$, affirment volontiers que le VIH n'est pas du tout visible sur leur corps, et leur score d'acceptation du VIH est meilleur encore que dans le groupe $G 1$. Mais ils rompent radicalement avec les répondants de $G l$ lorsqu'ils estiment, davantage que tous les autres interrogés, que leur «corps est mieux qu'avant ${ }^{14}$, et que le VIH a amélioré leur existence. Ce deuxième groupe se démarque aussi du premier par une plus forte divulgation de l'information sur sa séropositivité, fréquemment dite à plus de dix personnes, au conjoint et à plus de deux amis. Sans doute leur long passé de malades y est-il pour quelque chose. Enfin, G2 est composé de davantage de personnes qui fréquentent une association, avec ou sans lien avec le VIH.

\section{3-2-Les loisirs au regard de l'état de santé perçu}

\footnotetext{
${ }^{12}$ Variable «Quel est votre niveau d'acceptation de votre VIH ? de 1 : 'j'accepte très bien' à 5 : 'j'accepte très mal' » : prise comme variable nominale, on note la surreprésentation de la modalité «j'accepte très bien mon VIH » $(1 / 5)$ pour les répondants de $G 1$; prise comme variable d'échelle, leur score est significativement inférieur à la moyenne.

${ }^{13}$ Rappelons que ce groupe est marqué par une sur-déclaration de la stabilité du poids depuis le diagnostic.

${ }^{14}$ Avec un score d'adhésion beaucoup plus fort que tous les autres répondants, contrairement aux autres questions sur la modification du regard sur le corps.
} 
Alors que l'expérience du VIH de G1 (ASL stables) et G2 (ASL accrues) s'opposent au regard de la gestion de l'information sur la séropositivité (son invisibilisation permettant aux répondants de G1 d'assurer une continuité gage du maintien dans la normalité) -, un continuum de dégradation du vécu s'esquisse entre $G 3$ (ASL fluctuantes), G4 (ASL réduites) et $G 5$ (ASL interrompues). La baisse des ASL est ici en lien avec une détérioration de la perception de la situation socio-sanitaire.

Les répondants de $G 3$ affirment massivement une modification de leur regard sur leur corps. Leurs rapports au corps fluctuent entre l'affirmation «ce n'est pas moi ${ }^{15}$ et «mon corps est déformé » (avec un score au-dessus de la moyenne de la population). Ils estiment leur VIH fréquemment visible, voire très visible. Ils sont aussi plus enclins que la moyenne à affirmer se peser plus souvent qu'avant. Il faut rappeler que plus d'un quart d'entre eux déclarent que leur poids a beaucoup fluctué depuis le diagnostic.

Outre le sentiment d'avoir un corps transformé, ces répondants affirment plus volontiers que ceux de $G 1$ et $G 2$ que le VIH a un impact négatif sur leur vie et «qu'il complique singulièrement leur existence ». A l'inverse des deux groupes précédents, les répondants déclarant côtoyer une ou deux PVVIH par semaine sont surreprésentés. Enfin, plus encore que $G 2$, et contrairement à $G 1$, ces PVVIH aux ASL fluctuantes diffusent plus largement l'information sur leur statut sérologique, avec une surreprésentation des répondants l'ayant dit à plus de dix personnes, mais aussi une fréquence plus importante de la connaissance de la séropositivité par la mère, par les frères et sœurs, par les enfants, par plus de deux amis, et même par plus de deux collègues. Leurs nombreuses maladies, et leur volonté affichée de prendre activement en charge leur santé a dû y contribuer.

Les interrogés qui ont réduit leurs ASL $(G 4)$ affirment encore plus volontiers que ceux du groupe $G 3$ que le VIH a un effet négatif sur leur vie, soit qu'il «complique singulièrement leur existence », soit qu'il la rende « tout à fait insupportable ». Leur très mauvais score d'acceptation de leur $\mathrm{VIH}^{16}$ s'ajoute à une fréquence élevée des pensées à propos du VIH durant la semaine écoulée. L'idée d'une transformation du regard sur son corps depuis le diagnostic est par ailleurs largement partagée. Cette transformation ne peut pas être envisagée comme positive - comme au sein de $G 2-$, mais est nécessairement négative. Leur corps, déprécié («pas séduisant», «trop gros », «déformé »), est devenu un véritable alter ego dans lequel ils ne se reconnaissent pas ${ }^{17}$. Pas étonnant donc que leurs trajectoires professionnelles et leur moral apparaissent fortement affectés par le VIH, ou encore qu'ils indiquent ne plus aimer les activités physiques et sportives (alors qu'ils les aimaient avant le diagnostic), désormais associées à un risque d'usure ou de mal être.

La très forte appréhension du vieillissement avec le VIH est sur-déclarée (et le score moyen plus élevé que la moyenne), tout comme le fait de se regarder dans le miroir et de se peser «moins souvent qu'avant ». Les répondants de $G 4$ hésitent sur la question de la visibilité corporelle de leur VIH, affirmant plus souvent que les autres qu'il est «plutôt visible » ou «plutôt non visible ». Ils s'estiment cependant plus fréquemment marqués corporellement par le VIH que la moyenne de la population (score moyen supérieur). On note parmi eux la fréquentation élevée de plus de 5 PVVIH par semaine, et le fait d'avoir souvent évoqué la séropositivité avec toute ou partie de leur fratrie.

Enfin, les répondants de $G 5$ offrent les indicateurs les plus négatifs de vécu du VIH. Ils sont obnubilés par le VIH et l'acceptent très $\mathrm{mal}^{18}$ : il induit une existence «tout à fait insupportable ». Ce groupe est par ailleurs caractérisé par une très forte appréhension du vieillissement avec le virus. Or, ils prennent peu en charge leur santé. Leurs ressources culturelles, financières et sociales très faibles (liées notamment à la surreprésentation de migrants dans ce groupe) peuvent expliquer cela.

\footnotetext{
${ }_{15}$ Au sujet de la variable «Êtes-vous d'accord avec la phrase 'lorsque je vois mon corps, je me dis que ça n'est pas moi' ? », prise comme variable nominale, les répondants de $G 3$ adoptent fréquemment la réponse médiane.

${ }^{16}$ Lorsque la variable est prise comme variable nominale, surreprésentation des modalités « j'accepte mal/très mal mon $\mathrm{VIH} »(4$ et 5/5), et lorsque la variable est prise comme variable d'échelle, score bien supérieur à la moyenne.

${ }^{17}$ Plus de la moitié des répondants du G4 ne sont ainsi «pas du tout d'accord » avec l'idée que la proposition : «mon corps est mieux qu'avant », tandis que leurs scores moyens sont systématiquement plus élevés que la moyenne pour les autres propositions négatives sur leur corps.

${ }^{18}$ Prise comme variable nominale, choix significativement haut de $5 / 5$; prise comme variable d'échelle, score très fortement supérieur à la moyenne.
} 
Les répondants de $G 5$ déclarent massivement une modification du regard sur leur corps, qu'il n'est pas séduisant, qu'il est déformé, qu'ils ne se reconnaissent pas en lui (scores les plus élevés de la population pour ces trois items, et le moins élevé pour l'item «corps mieux qu'avant»). Tout comme ceux de G4, ils déclarent moins volontiers se regarder dans le miroir et se peser qu'avant, et perçoivent les APS comme facteur d'inquiétudes, un risque d'usure ou de mal être ${ }^{19}$. A la différence de $G 4$, on note cependant ici une plus forte fréquence de ceux qui n'ont pas informé leurs collègues de leur séropositivité, ni même leur partenaire : malgré leur vécu très dégradé, ils se rapprochent sur ce point de Gl.

\section{3-3- Les « loisirs » associatifs : entre prescription et fréquentation des marges}

Alors que pour les PVVIH qui fréquentent d'autres types d'associations, les ASL apparaissent surtout comme une façon de signifier le maintien dans une normalité sourdement menacée, celles qui fréquentent les associations VIH les vivent davantage comme un baromètre de leur état de santé (cf. encadré $n^{\circ} 4$ ). L'inscription dans ces dernières semble ainsi contribuer à l'établissement d'un lien entre santé et loisirs inhérent au statut de malade chronique, comme malade acteur. Ce lien n'empêche toutefois pas une grande diversité des rapports aux loisirs, les personnes acceptant ou résistant plus ou moins au statut qui leur est proposé.

\section{Encadré $\mathrm{n}^{\circ} 4$ : Préoccupations sanitaires et recours aux ASL des associations VIH}

La trajectoire de Christian, 48 ans, investi dans un des créneaux d'activités physiques mis en place au sein d'une association de services observée, illustre les perturbations des ASL après le diagnostic. Danseur professionnel, il évoque une phase de dépression juste à la suite de celui-ci, où il stoppe toute pratique. Puis, progressivement, il intègre les créneaux d'activité physique adaptée d'une association VIH, tout en poursuivant une activité physique d'entretien à domicile. Sa pratique prend alors une nouvelle signification, liée à son anticipation des effets secondaires des traitements : «Donc c'était beaucoup plus ciblé ce que je faisais pour contrer les effets ». Il précise : «Le fait d'être séropositif m'a motivé en fait en moi à faire du sport, plus que je ne le faisais avant », avant d'ajouter : "On sait que quand on prend des médicaments, il faut s'attaquer aux fesses, aux abdos, tout ça ». Le sport, perçu comme un «accompagnement de la trithérapie », devient incontournable. L'engagement associatif lui permet d'avoir un repère concernant les modifications corporelles qu'il vit, en l'aidant « à comprendre comment les corps des autres évoluent par rapport à la maladie ».

Dans les associations VIH observées, les individus recourent à un certain nombre d'adaptations primaires pour adhérer aux «bons » loisirs proposés. Mais ils déploient aussi des adaptations secondaires pour construire des formes de loisir à la marge. Les observations montrent ainsi que si certains participants se conforment totalement au rôle attendu (celui du «bon » malade qui fait du sport, mange «bien », dort «bien », ne fume pas, est attentif et apprend), d'autres ne se conforment que partiellement à ces prescriptions. Ces derniers mettent en place des actions (discuter, rire, se bagarrer durant un atelier d'activité physique, boire de l'alcool, draguer) «déviantes », au sens de Becker, dans la mesure où elles sont sanctionnées par des remarques de l'intervenant visant à les proscrire. Si certaines de ces pratiques à la marge peuvent se dérouler durant les activités associatives, d'autres sont organisées dans des lieux et temps différents. Ainsi, un groupe de «bénéficiaires » s'auto-organise pour faire des week-ends de «relaxation » où tous les usages considérés comme déviants dans le cadre associatif sont autorisés, voire encouragés :

\footnotetext{
${ }^{19}$ Comme G4, certains disent ne plus aimer les activités physiques et sportives depuis le diagnostic ; ils sont toutefois également nombreux à affirmer qu'ils ne les ont jamais aimées.
} 
consommation d'alcool, de marijuana, de tabac, comportement de séduction, rires, discussions non liées à la prise en charge, nuits blanches.

Si l'ensemble des «usagers » associatifs ont des profils similaires ${ }^{20}$, ceux qui s'engagent dans ces activités déviantes sont caractérisées par leur ancienneté dans les associations. En fournissant un lieu de regroupement, ces dernières permettent ici la reconstruction d'espaces de loisirs libérés des contraintes qu'elles tentent pourtant par ailleurs d'imposer : si on y apprend les attentes sociales à l'égard du «bon malade », on y apprend aussi à se distancier de ces normes en participant à des actes déviants. On pourrait ainsi s'attendre, avec cette prise de distance, et la participation à des ASL entre pairs hors du cadre établi, à un départ progressif des associations. L'une d'elle en fait d'ailleurs l'un de ses principaux objectifs, se voulant un espace «intermédiaire entre la prise en charge et le loisir ${ }^{21}$. Confrontée à un succès grandissant, et ne pouvant plus accueillir de nouveaux «bénéficiaires », elle tente d'orienter les « usagers » perçus comme n'ayant plus besoin des ateliers qu'elle propose vers des structures « ordinaires »... en vain.

Il semble donc difficile aux «bénéficiaires » de quitter des espaces pourtant contraignants, et au sein desquels ils sont parfois considérés comme plus ou moins « déviants », pour s'insérer dans des ASL «normales ». Faut-il voir là l'effet d'une volonté de se préserver de la stigmatisation, ou bien une impossibilité de refuser le rôle de «bon malade » et certains de ses bénéfices ? On peut se demander si, au final, cette population captive des associations ne peut envisager d'avoir des loisirs « normaux » qu'en contrepartie d'un engagement dans des loisirs « de prise en charge »; et comme une juste récompense de leurs efforts. La culpabilité induite par la résistance à l'égard des normes de prise en charge imposées serait ainsi un frein au plaisir généré par le loisir «normal ». Pour certains, le rapport aux loisirs paraît se construire uniquement en marge de l'obligation des loisirs de « prise en charge ».

\section{4- Les loisirs post-diagnostic : besoin de normalité et/ou indicateur de bien-être social ?}

Les résultats présentés restent évidemment limités sur le plan méthodologique. L'exploitation $\mathrm{du}$ questionnaire repose sur une question unique qui renvoie à une posture déclarative sur l'évolution des ASL depuis le diagnostic (et ne dit pas grand-chose sur l'éventuelle évolution des usages) introduit un biais rétrospectif. De même, le nombre d'associations observées est réduit et limité au fonctionnement du milieu de la lutte contre le VIH dans une région de France.

En resituant l'évolution des expériences de loisir post-diagnostic VIH au sein des trajectoires sanitaires et sociales, l'étude fait néanmoins apparaître deux processus qui pèsent sur les modalités d'engagements des PVVIH : un maintien dans la normalité où les loisirs sont un signe de continuité de l'existence ; une prise en charge de soi où les loisirs tendent à s'appauvrir et à se transformer en obligation sanitaire liée à la carrière de malade chronique. Pour l'essentiel, si l'on excepte le cas spécifique de G2 (ASL accrues), ces deux processus agissent en sens inverse selon un continuum de dégradation de la perception de sa situation sanitaire qui est en rapport avec sa situation sociale générale et le fait, entre autres, de maintenir une activité professionnelle ou de de fréquenter des associations VIH :

Perception de sa situation sanitaire et social + Loisirs inchangés, signe de normalité Activité professionnelle

Perception de sa situation sanitaire et sociale -

............................. Moins de loisirs, demande d'aides

Les cas spécifiques de $G 2$ et $G 3$ sont ici particulièrement intéressants puisque, malgré un impact plus négatif du VIH au plan strictement sanitaire que pour $G 4$ et $G 5$, ils le vivent mieux. Le loisir peut-il pour autant être considéré comme un indicateur de bien-être social, sinon de qualité de vie ? La relation ne paraît ici pas si simple. Chacun le revendiquant comme un signe de normalité,

\footnotetext{
${ }^{20}$ Ce sont en général des gens de plus de 40 ans, sans profession, avec de faibles revenus, anciennement diagnostiquées.

${ }^{21}$ Propos tenu par un dirigeant lors d'une réunion d'accueil des bénéficiaires.
} 
ne devient-il pas une nécessité et un vecteur d'intégration sociale, a fortiori pour les personnes confrontée à un risque d'exclusion sociale ? Pour les individus inscrits dans les systèmes de prise en charge médico-sociale, son instrumentalisation conduit en outre à en faire, non plus un indicateur ou un facteur de bien-être, mais un outil de préservation de la qualité de vie et de sa santé. Naît ainsi le paradoxe de loisirs «adaptés » et «contraints », dont l'organisation renvoie aux devoirs, sinon aux prescriptions, d'une carrière de malade.

Le VIH confronte à une alternative : maintenir une continuité synonyme de normalité (par rapport à la norme de son existence d'avant), ou repenser ses activités à partir d'une réorganisation de son quotidien. Pour ceux qui ont une activité professionnelle, la normalité sociale s'inscrit d'abord dans le maintien de l'alternance entre temps de travail et temps libéré du travail. Parallèlement à la carrière professionnelle, une autre carrière s'ouvre, avec ses contraintes spécifiques (liées à l'observance thérapeutique et à la prise en charge de soi). Bien sûr, le désir de normalité conduit à occulter ces contraintes, pour faire comme si rien n'avait changé. Il n'est pas question de s'adapter, mais de continuer comme avant, quels qu'en soient les risques.

Lorsque la carrière professionnelle fait défaut, ou qu'elle doit s'interrompre, il faut reconfigurer son temps. L'aide des associations VIH est précieuse. Elle impose toutefois une contrepartie : embrasser la carrière de malade chronique, comme un devoir imposé par des entrepreneurs de morale (Becker, 1963). Dans ce cadre, les loisirs comportent leur dose de contrainte, dont il n'est possible de se libérer qu'à la marge. Les personnes s'efforcent bien sûr de reconstruire des utilités marginales ou des rendements marginaux de loisir (Yonnet, 1999) dans le temps contraint de la «bonne » gestion de soi et de sa maladie. L'entreprise n'est pas facile, et relève d'adaptations secondaires (Goffman, 1968). Au final, la fuite de ce temps associatif rempli d'obligations ne peut s'opérer que dans le désordre de « rituels inversés » (Balandier, 1988) qui dévoilent, en miroir, les exigences pesantes de la carrière de «malade chronique ».

Les enjeux liés à l'expérience des loisirs suite au diagnostic de VIH n'apparaissent jamais aussi clairement que dans les questions générées par l'accès aux activités physiques et sportives. A l'heure où les loisirs sportifs apparaissent comme un espace de créativité offrant des signes d'intégration et de normalité sociale (Corneloup \& Mao, 2010; Ferréol, 1999), l'ensemble de la littérature biomédicale et de l'offre proposée par les associations VIH les envisagent d'abord comme des outils d'intervention médico-sociale (Ferez et al., 2010 ; Ferez \& Luauté, 2008). Dans ce cadre, la pratique physique et sportive est perçue comme nécessairement «bonne » et en mesure de réduire les "situations de handicap », en générant un accroissement des capacités de contrôle de son existence (O'Brien et al., 2009). La réalité du vécu des PVVIH atteste pourtant, à l'inverse, d'une pratique à la fois hantée par la crainte de l'érosion de ses capacités et par la peur de la visibilité de «son»VIH (Ferez \& Thomas, 2012). Face à cette expérience fragile, des dispositifs inclusifs peuvent-ils être pensés hors d'une logique de diffusion des « bonnes » pratiques? 


\section{Bibliographie}

Adams, P. et C. Herzlich (1994). Sociologie de la maladie et de la médecine. Paris : Nathan.

Balandier, G. (1988). Le Désordre : éloge du mouvement. Paris : Fayard.

Becker, H. (1963). Outsiders. Etudes de sociologie de la déviance. Paris: Métailié.

Bouvier, P. (2005). Le Lien social. Paris : Gallimard. 2005.

Bury, M. (1982). Chronic Illness as Biographical Disruption. Sociology of health \& illness, 4(2), 167-182.

Cibois, P. (1993). Le PEM, pourcentage de l'écart maximum : un indice de liaison entre modalités d'un tableau de contingence. Bulletin de méthodologie sociologique, 40, 43-63.

Corbin, A. (1995 [2001]). L'Avènement des loisirs, 1850-1960. Paris : Flammarion/Champs.

Corbin, J. M. et A. L. Strauss (1991). A Nursing Model for Chronic Illness Management based upon the Trajectory Framework. Scholarly Inquiry for Nursing Practice, 5(3), 155-174.

Corneloup, J. et P. Mao (dir.) (2010). Créativité et innovation dans les loisirs sportifs de nature. Un autre monde en émergence. Paris : Editions du Fournel.

Dumazedier, J. (1962). Vers une civilisation du loisir?. Paris : Seuil.

Dumazedier, J. (1988). Révolution culturelle du temps libre, 1968-1988. Paris : Klincksieck.

Duval, E., Liotard, P. et J. Thomas (2012). Activités physiques, couple et prise en charge du VIH: de la facilitation à la contrainte face au diagnostic. In S. Ferez et J. Thomas (dir.), Sport et VIH. Paris: Téraèdre.

Ferez, S. et J.-P. Luauté (2008). L'activité physique et sportive comme outil médicopsychologique? Etude de l'offre de pratiques en direction des personnes infectées par le VIH. Annales médico-psychologiques, 166(10), 847-849.

Ferez, S., Athanasios, P. et S. Ruffié (2010). La actividad física y deportiva de las PVVIH : Del interés por la práctica al acceso a la práctica (revisión de la bibliografía). Retos: nuevas tendencias en educación física, deporte y recreación, 18, 77-83.

Ferez, S. et J. Thomas (2012). Sport et VIH, un corps sous contrainte médicale. Paris: Téraèdre.

Ferréol, G. (dir.) (2009). Représentations corporelles et loisirs sportifs. Fernelmont: Éditions modulaires européennes.

Fougeyrollas, P. (2010). Le funambule : le fil et la toile, transformations réciproques du sens du handicap. Laval : Presses Universitaires de Laval.

Goffman, E. (1961 [1968]). La vie clandestine d'une institution totalitaire. In E. Goffman, Asiles, études sur la condition sociale des malades mentaux et autres reclus. Paris : Minuit.

Haddad, B. (2011). Religion and HIV and AIDS: Charting the terrain. Scottsville, South Africa: University of ZwaZulu-Natal Press.

Hurley, M. (2010). VIH, santé sexuelle et cultures gaies contemporaines. In O. Jablonski, J.Y. Le Talec et G. Sidéris (dir.), Santé gaie. Paris : L'Harmattan/Editions Pepper : 117-142.

Jablonski, O., Le Talec.Y. et G. Sidéris (dir.) (2010). Santé gaie. Paris : L’Harmattan/Editions Pepper.

Langlois, E. (2006). L'épreuve du sida. Pour une sociologie du sujet fragile. Rennes : Presses Universitaires de Rennes.

Le Breton, D. (1998). La contamination du sens. Ethnologie française, XXVII(1), 37-42.

Lemelin, A. (2004). Méthodes quantitatives des sciences sociales appliquées aux études urbaines et régionales. Montréal : INRS-UCS.

Mellini, L., Godenzi, A. et J. De Puy (2004). Le Sida ne se dit pas. Analyse des formes de secret autour du VIH/sida. Paris : L'Harmattan.

Memmi, D. \& D. Fassin (2004). Le gouvernement des corps. Paris: Editions EHESS.

O’Brien, K. K., Davis, M. A., Strike, C., Young, N. L. et A. M. Bayoumi (2009). Putting Episodic Disability into Context: a Qualitative Study Exploring Factors that Influence Disability Experienced by Adults Living with HIV/AIDS. Journal of International AIDS Society, 12, 30-41. 
Pierret, J. (1997). Un objet pour la sociologie de la maladie chronique : la situation de séropositivité au VIH ?. Sciences sociales et santé, 15(4), 97-120.

Pierret, J. (2006). Vivre avec le VIH. Enquête de longue durée auprès des personnes infectées. Paris : Presses Universitaires de France.

Pronovost, G. (2002). Les temps sociaux : une comparaison France-Canada-Québec. In A. Huet et G. Saez (dir.), Le Règne des loisirs. Paris : Datar/Editions de 1'aube, 41-68.

Saez, G. (2002). Les loisirs, temps social, temps pour soi, temps aménagé. In A. Huet et G. Saez (dir.), Le Règne des loisirs. Paris : Datar/Editions de l'aube, 11-39.

Teboul, R. (2004). Culture et loisirs dans la société du temps libre. Paris : Editions de l'aube. 